\title{
ADDITION OF A SUBJECTIVE NURSING ASSESSMENT IMPROVES SPECIFICITY OF A TOOL TO PREDICT ADMISSION OF CHILDREN TO HOSPITAL FROM AN EMERGENCY DEPARTMENT
}

\author{
D. Roland ${ }^{1}$, G. Lewis ${ }^{2}$, F. Davies ${ }^{2}$, Paediatric Emergency Medicine Leicester Academic Group \\ ${ }^{I}$ Cardiovascular Sciences, University of Leicester, ${ }^{2}$ University Hospitals of Leicester NHS Trust, Leicester, \\ $U K$
}

Background: Children with serious illness can be difficult to spot, especially for non-experienced staff. Although adult "early warning scores" are now commonly used in Emergency Departments (ED), there is no such system for children. The Paediatric Observation Priority Score (POPS) is a physiological and observational scoring system (Range $0-16$ ) designed for use by health care professionals of varying clinical experience at initial assessment in our ED.

This phase of our validation examined the utility of the more subjective criteria of POPS (level of alertness, work of breathing, nursing concern and relevant background history) against the physiological criteria (heart rate, breathing rate, saturations and temperature) in determining admission to the children's hospital assessment unit from the ED.

Methods: Prospective casenote review of attendances to the ED, analysing patient discharge disposition against initial POPS recorded.

Results: 942 (injuries not included) presentations were included in the study with an overall admission rate of $36 \%$ (339/942). Relative risk of admission of admission with a POPS of $>1$ was 2.3 (1.9-2.8). Further results are shown in Table One.

\begin{tabular}{|l|c|c|}
\hline \multicolumn{1}{|c|}{} & Specificity & Sensitivity \\
\hline Objective Criteria & 0.72 & 0.56 \\
\hline Subjective Criteria & 0.92 & 0.26 \\
\hline [Table One]
\end{tabular}

Conclusion: Subjective nursing concern at initial assessment is a specific but poorly sensitive methodology of predicting admission. This complexity may prohibit its wide-scale use in this role in Emergency Departments however its use as resource management tool is an area we are currently investigating. 